\title{
Complex environmental $\beta$-plane turbulence: laboratory experiments with altimetric imaging velocimetry
}

\author{
A. M. Matulka, Y. Zhang, and Y. D. Afanasyev \\ Memorial University of Newfoundland, St. John's, Canada \\ Correspondence to: Y. D. Afanasyev (afanai@mun.ca)
}

Received: 9 October 2015 - Published in Nonlin. Processes Geophys. Discuss.: 9 November 2015

Revised: 8 January 2016 - Accepted: 12 January 2016 - Published: 28 January 2016

\begin{abstract}
Results from the spectral analyses of the flows in two experiments where turbulent flows were generated in a rotating tank with a topographic $\beta$-effect are presented. The flows were forced either by heating water from below or supplying fresh water at the top of a saline layer. The flow was essentially barotropic in the first experiment and baroclinic in the second experiment. The gradient of the surface elevation was measured using optical altimetry (altimetric imaging velocimetry). Multiple zonal jets of alternating direction were observed in both experiments. Turbulent cascades of energy exhibit certain universal properties in spite of the different natures of flows in the experiments.
\end{abstract}

\section{Introduction}

Two-dimensional $\beta$-plane turbulence is an important concept in complex environmental flows where planetary rotation and the effect of the variation of the Coriolis parameter with latitude ( $\beta$-effect) are significant factors. Kraichnan (1967) formulated a Kolmogorov-type theory which predicted the slope $-5 / 3$ of the energy spectrum in the energy range and the slope -3 in the enstrophy range for two-dimensional turbulence. The $\beta$-effect modifies two-dimensional turbulence towards anisotropy. The energy spectra in wavenumber space become a figure-of-eight with most of the energy concentrated at a zonal wavenumber close to zero. In physical space this effect manifests itself in the creation of zonal jets. Oceanographic observations of this phenomenon have been extensively discussed in the literature (Maximenko et al., 2005, 2008; Centurioni at al., 2008; Ivanov et al., 2009).

Experiments on the Coriolis rotating platform by Read et al. (2007) confirmed the theoretical prediction of a
$-5 / 3$ slope in the energy range. The authors used convective forcing in their experiments which generated motions of very small scale. Since the size of the domain was large, the scale separation was large enough for the development of the inverse cascade of energy. A recent study on two-dimensional turbulence (in the absence of $\beta$-effect) by Afanasyev and Craig (2013) gave the experimental evidence of dual cascade with the spectral slopes of $-5 / 3$ and -3 . Further experiments with barotropic turbulence on the $\beta$-plane by Zhang and Afanasyev (2014) demonstrated the dual cascade in the presence of $\beta$-effect as well as the figure-of-eight energy spectrum in the wavenumber space.

Zonal jets have a long history of investigation starting from the pioneering experiments by Whitehead (1975) and Collin de Verdiere (1979). The jets readily form when a spatially localised forcing is applied in the $\beta$-plane fluid (e.g. Sommeria et al., 1988, 1989; Marcus and Lee, 1998; Afanasyev et al., 2011; Slavin and Afanasyev, 2012). However, a distributed forcing such as that provided by baroclinic instability also creates jets. In the ocean, the regions where baroclinic instability is dynamically important include the Antarctic Circumpolar Current (ACC) as well as western boundary currents and their extensions. Multiple jets as well as mesoscale eddies are created there by the baroclinic instability, thus forming a dynamically complex turbulent flow. A classic model for a baroclinically unstable system is a rotating annulus where heating/cooling is provided at the outer wall/centre of the tank respectively. Different aspects of the dynamics of this system were studied in a number of experiments (Hide and Mason, 1975; Mason, 1975; Bastin and Read, 1997, 1998; Wordsworth et al., 2008; Smith et al., 2014). A somewhat different experimental approach to modelling a baroclinically unstable two-layer flow with vertical shear was 
used in a recent study by Matulka and Afanasyev (2015). It was shown that the meridional scale of the jets is determined to a large extent by the radius of deformation and, at the same time, is in good agreement with the Rhines scale (Rhines, 1975). The jets are driven by (non-linear) Reynolds stresses due to baroclinic meanders. This is in agreement with a scenario described by Berloff et al. (2009a, b). The authors describe the formation of jets as a secondary instability of the primary instability of the baroclinic flow in the form of mainly meridional motions (so-called "noodles").

In this study we perform spectral analyses of the flows described in Matulka and Afanasyev (2015) and compare them with the results obtained for a somewhat different flow generated by thermal forcing (Zhang and Afanasyev, 2014). The latter experiment although forced baroclinically was more barotropic in its dynamics, while the former experiment was purely baroclinic. In Sect. 2 of this paper, we describe the laboratory set-up for both experiments. In Sect. 3 the results of the spectral analyses are reported. Concluding remarks are made in Sect. 4.

\section{Experimental technique}

The laboratory experiments were carried out in a cylindrical tank of radius $R=55 \mathrm{~cm}$ filled with water of depth $H_{0}=10$ $12 \mathrm{~cm}$. The tank was installed on a rotating table (Fig. 1a) and rotated in an anticlockwise direction at a constant angular rate $\Omega=2.32 \mathrm{rad} \mathrm{s}^{-1}$. In this paper we compare two experiments where the flows were forced in two different ways. The first experiment was forced thermally with a heating wire at the bottom of the tank. The wire was arranged in an approximately uniform pattern such that the distance between the segments of the wire was $4.5 \mathrm{~cm}$. Figure $1 \mathrm{~b}$ shows the magnitude of velocity in the range between 0 (black) and $0.5 \mathrm{~cm} \mathrm{~s}^{-1}$ (white) at the very beginning of the experiment. The flow is initially along the wires such that the wire pattern is visible. This wire arrangement (in contrast to, say, a spiral) was chosen in order to avoid a direct forcing in the zonal direction but yet to provide an approximately uniform mean heat flux across the bottom. The heater supplied the total power of $2300 \mathrm{~W}$.

In the second experiment the flow was forced by delivering fresh water at the surface of a salt water layer of salinity $S=30$ ppt. The fresh water source was distributed along the wall of the tank and created a coastal current flowing anticlockwise around the tank. The fresh water was pumped into the tank by a pump which delivered $20 \mathrm{~L}$ of water in about $6 \mathrm{~min}$. Figure 1c shows the camera view of the flow in the middle of the forcing period when the fresh water from the source has not yet spread over the entire surface of the tank but is concentrated mainly in the coastal current.
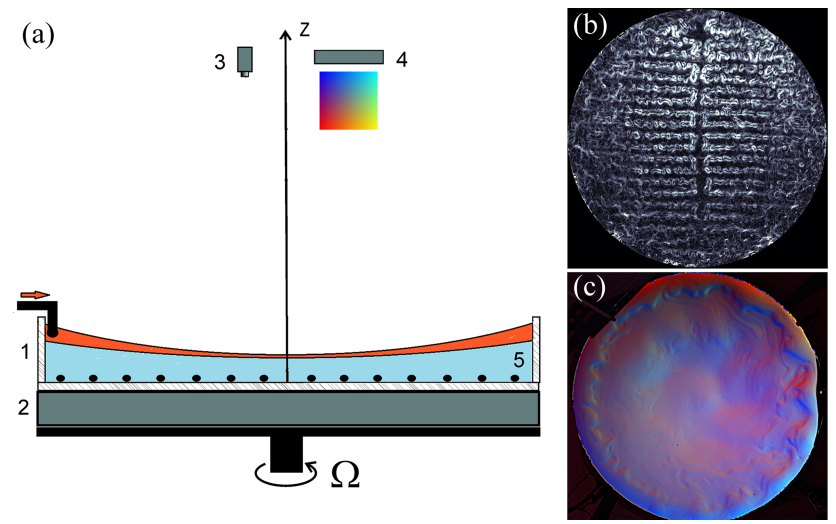

Figure 1. Sketch of the experimental set-up (a) and view of the flow at the beginning of the experiments with the thermal (b) and saline (c) forcing: (1) cylindrical tank filled with water and installed on a table rotating with angular velocity $\Omega$; (2) light box for the optical thickness measurements; (3) video camera; (4) light source with colour mask; and (5) heating wire on the bottom.

The free surface of the rotating fluid is a paraboloid when in solid-body rotation. The height of the water surface varies quadratically with the distance $r$ from the axis of rotation

$h(r)=H_{0}+\frac{\Omega^{2}}{2 g}\left(r^{2}-\frac{R^{2}}{2}\right)$,

where $g$ is the gravitational acceleration. This creates a topographic (polar) $\beta$-plane where the $\beta$-parameter at some distance $r=r_{0}$ from the pole is given by

$\beta=\left.\frac{f_{0}}{h(r)} \frac{\mathrm{d} h(r)}{\mathrm{d} r}\right|_{r=r_{0}}$,

where $f_{0}=2 \Omega$ is the Coriolis parameter. The values of the $\beta$-parameter in our experiments were between 0.07 and $0.1 \mathrm{~cm}^{-1} \mathrm{~s}^{-1}$.

We use the Altimetric Imaging Velocimetry (AIV) system to measure the gradient of the surface elevation $\eta$ (for more details see Afanasyev et al., 2009). AIV is based on optical altimetry first described in Rhines et al. (2006). Laboratory altimetry is not unlike the satellite altimetry which became an irreplaceable tool in oceanography. Apart from measuring $\nabla \eta$, AIV can be used as a tool to visualise the entire surface of the rotating fluid just like satellite altimetry provides a global coverage of the Earth's oceans. AIV also provides an alternative to the well-known particle imaging velocimetry (PIV) technique. To obtain the velocity field using PIV one has to find correlations between small areas (typically 12-48 pixels in both dimensions) of two successive images of the flow. If a camera has an imaging array of size say $1000 \times 1000$ pixels, the resulting array of velocity vectors has dimensions less than $100 \times 100$. Thus, the PIV technique effectively reduces the spatial resolution by a factor of 100 or more. AIV, on the other hand, allows one to obtain the 
velocity vector in every pixel of the image, which makes its spatial resolution practically unlimited given that the cameras with large imaging arrays are now easily available. The limitation of the AIV is that it can only be used in a relatively fast rotating fluid with free surface. It is, however, perfectly suited for oceanographic fluid dynamics experiments on the $\beta$-plane such as those described in this paper. The paraboloidal surface of the rotating fluid is used like the mirror of a Newtonian telescope. If the surface is disturbed by the pressure perturbations due to the flow, the slope of the surface changes slightly. These perturbations of the slope are detected by the AIV and measured using simple geometry and a colour coding.

AIV measures an "exact" (within experimental accuracy) surface elevation gradient, $\nabla \eta$, which translates into the pressure gradient, $\nabla p=\rho g \nabla \eta$, at the surface. Note that the main uncertainty is due to the colour noise of the camera sensor. To reduce the noise we pass data through a median filter with a window size of $5 \times 5$ pixels (physical size of approximately $0.2 \times 0.2 \mathrm{~cm}$ ). The overall uncertainty of $\nabla \eta$ can be estimated to be approximately $5 \%$. The velocity field is not measured directly by the AIV but is rather obtained from the measured $\nabla \eta$ using a (quasi-)geostrophic approximation.

The barotropic component of velocity can be calculated in geostrophic approximation as follows:

$\mathbf{V}=\frac{g}{f_{0}}(\boldsymbol{n} \times \nabla \eta)$

where $\boldsymbol{n}$ is the vertical unit vector. A next order of approximation is provided by the quasi-geostrophy, which gives

$\mathbf{V}=\frac{g}{f_{0}}(\boldsymbol{n} \times \nabla \eta)-\frac{g}{f_{0}^{2}} \frac{\partial}{\partial t} \nabla \eta-\frac{g^{2}}{f_{0}^{3}} J(\eta, \nabla \eta)$,

where $J$ is the Jacobian operator. The second and third terms on the RHS of Eq. (4) are corrections to the geostrophic velocity which take into account transient and non-linear effects. Their relative importance is determined by the temporal Rossby number $R o_{\mathrm{T}}=1 /\left(f_{0} T\right)$ and the Rossby number $R o=U /\left(f_{0} L\right)$ respectively. Here $T$ is the timescale of the unsteady processes in the flow, while $U$ and $L$ are velocity and length scales of the flow. Thus, the velocity field is determined more accurately when the flow is closer to being quasi-geostrophic. "Textbook" theory on the validity of the quasi-geostrophic approximation applies here. Since the Rossby number did not exceed unity even in the core of the eddies in our experiments and the mean values of the Rossby number were of the order of $10^{-1}$, the velocity was, on average, within $10 \%$ of the "exact" velocity.

Relative vorticity, $\zeta=\boldsymbol{n} \cdot \operatorname{curl} V$, was calculated by differentiating the velocity field. Since numerical differentiation amplifies noise in the original data, we used the Sobel gradient operators with $5 \times 5$ kernels (e.g. Pratt, 2007). The kernels are convolved with the velocity data to calculate the derivatives in $x$ and $y$ directions.

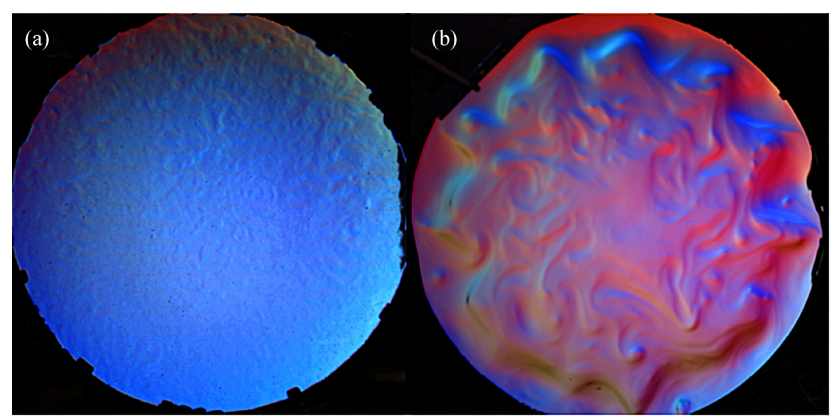

Figure 2. Typical images from video sequences recorded in the experiments with thermal (a) and saline (b) forcing. The flows are visualised by optical altimetry (AIV); different colours indicate different values (both in magnitude and direction) of the gradient of the surface elevation

According to the Taylor-Proudman theorem, the surface velocity given by Eq. (4) is a good approximation for the velocity in the entire column of water except the Ekman layer at the bottom. Note that in a stratified fluid, as in our two-layer experiment with saline forcing, the velocity field obtained by altimetry is in fact the barotropic component of the total velocity in the entire layer of water. It is also the upper layer velocity. A baroclinic component which allows one to obtain the total velocity in the lower layer can be measured by a different technique (e.g. Afanasyev et al., 2009; Matulka and Afanasyev, 2015), but is not discussed here.

\section{Results}

We performed two sets of experiments with different forcing, namely the thermal forcing by a wire heater at the bottom and the saline forcing by injection of fresh water at the wall. In what follows we discuss them in parallel, highlighting the similarities and differences between them.

\subsection{General flow evolution}

Figure 2a shows a typical snapshot of the surface of water in the experiment with the thermal forcing when the flow is fully developed. The flow is visualised by the AIV technique such that colour shows the horizontal gradient of the surface elevation, $\nabla \eta$. The colour intensity indicates the magnitude of $\nabla \eta$, while hue shows its direction. The motions are initially of very small scale in this experiment (see also Fig. 1b). Warm water heated by the wire rises to the surface in thin sheets and forms long thin filaments. These filaments are unstable with respect to baroclinic or frontal instability and break into small eddies. The size of the eddies is most likely determined by the baroclinic radius of deformation which can be defined as $R_{\mathrm{d}}=\left(g^{\prime} H_{0} / 2\right)^{1 / 2} /\left(g^{\prime} H_{0} / 2\right)^{1 / 2} f_{0}$, where $g^{\prime}$ is the reduced gravity in the warm water filaments and eddies. The reduced gravity is defined by the temperature 
difference between the water in filaments and background temperature of the water in the tank. However, since we did not measure the temperature field in this experiment, we cannot pinpoint the exact value of $R_{\mathrm{d}}$. It is important to establish here the values of control parameters for the thermal convection in this experiment. Flows created by buoyancy sources in the rotating fluid (in the absence of the $\beta$-effect) were studied in laboratory experiments by Fernando et al. (1991) and Maxworthy and Narimusa (1994). The energy flux per unit area of the bottom of the tank is $Q=2.5 \times 10^{3} \mathrm{Wm}^{-2}$ in our experiments. This translates into the buoyancy flux $B=\alpha g Q / C_{\mathrm{p}}=1.2 \times 10^{-6} \mathrm{~m}^{2} \mathrm{~s}^{-3}$, where $\alpha$ is the thermal expansion coefficient and $C_{\mathrm{p}}$ is the volumetric heat capacity of water at constant pressure. A dimensionless parameter which controls the regime of the thermal convection is the Rayleigh number. The flux Rayleigh number is quite high in our experiments, $R a_{\text {flux }}=B H_{0}^{4} / \nu^{2} \kappa=1.8 \times 10^{9}$ (where $\kappa$ is the thermal diffusivity of water), which indicates a regime of turbulent convection. Comparing to the recent experiments by Read et al. (2015) where similar heating was used but in an experiment of a larger scale, we note that our buoyancy flux was almost 2 orders of magnitude higher; the Rayleigh number was, however, an order of magnitude lower because of the smaller depth of water in our experiments.

Figure $3 \mathrm{a}$ and $\mathrm{b}$ shows the fields obtained as a result of the velocity calculation from measured $\nabla \eta$ in the thermal experiment. Azimuthal velocity $V_{\mathrm{az}}$ in a polar coordinate system with the origin at the centre of the tank is shown in panel a, while panel b shows the relative vorticity, $\zeta=\boldsymbol{n} \cdot \operatorname{curl} \mathbf{V}$. The relative vorticity field shows a fine structure of the flow with multiple small-scale cyclonic (red) and anticyclonic (blue) eddies. The relative vorticity is normalised by the Coriolis parameter $f_{0}$; thus, the image in panel $\mathrm{b}$ can be interpreted as a map of the Rossby number, $R o=\zeta / f_{0}$. The values of $R o$ can reach unity in the strongest eddies, while the rms value is approximately 0.2. An original alignment of filaments with the heating wires can still be seen in the central area of the tank, while closer to the wall where the water is deeper and the $\beta$-effect is stronger, the bands of positive and negative vorticity are aligned in the zonal direction, which indicates the presence of zonal jets. The jets can be seen more clearly in the azimuthal velocity image in Fig. 3a. The circulation is mainly in the anticlockwise (eastward) direction ( $V_{\mathrm{az}}$ is positive, red colour). Maximum values of $V_{\mathrm{az}}$ in the jets are approximately $1 \mathrm{~cm} \mathrm{~s}^{-1}$.

Figures $2 b$ and $3 c$ and d (see also Fig. 1c) show the experiment with the saline forcing. The water in the tank is initially of salinity $S=30 \mathrm{ppt}$. When a source distributed along the wall of the tank delivers fresh water, it creates a current along the wall. This current is initially wedge-shaped in cross section and is approximately in geostrophic balance such that it "leans" on the wall to its right. This coastal current can be seen clearly in Figs. 1c, $2 b$ and $3 c$; the velocity of the current is initially in excess of $5 \mathrm{~cm} \mathrm{~s}^{-1}$. The current is baroclinically unstable (e.g. Griffiths and Linden, 1981) and creates meanders which penetrate into the interior of the tank. During the forcing period of the experiment when the source continuously supplies fresh water, the entire surface of the tank eventually becomes covered with a layer of fresh water. Thus, a two-layer system is created. The forcing then stops and the flow is allowed to develop freely. The depth of the upper layer is not uniform in the radial direction after the period of forcing. The layer is much thicker at the wall of the tank rather than at its centre. Thus, the system contains a large amount of available potential energy which is released gradually and maintains the flow for a very long time after the forcing stops. The adjustment involves slow radial motion towards the centre in the upper layer and the motion in the opposite direction in the lower layer. The radial motion, in turn, causes zonal circulation. Measurements of barotropic and baroclinic components of velocity (for details see Matulka and Afanasyev, 2015) show that the upper layer rotates cyclonically while the lower layer rotates anticyclonically. The shear between the layers makes the system baroclinically unstable. Conditions for the development of the baroclinic instability are maintained over a long period of adjustment. Note that measurements of mean energy and enstrophy of the system (not shown here) during the long period of adjustment indicate that the system is approximately steady in this experiment.

Baroclinic instability together with other instabilities including, perhaps, wave breaking and barotropic and frontal instabilities, continuously generates meanders over the entire area of the tank. The meanders move water parcels in the radial (meridional) direction. According to conservation of potential vorticity the parcels acquire additional relative vorticity and radiate Rossby waves. Motion of the meanders/parcels correlated via the global Rossby wave field creates the Reynolds stresses which drive zonal jets in the interior of the tank. Thus, although direct forcing was stopped, the system is forced by the baroclinic instability similar to that in the ocean. Measurements of the Reynolds stresses in this experiment showed that jets in the interior are dynamically different from the coastal jet which is affected by the presence of the wall. The jets in the interior are true eddyforced jets, while the coastal current is not. In what follows we perform spectral analyses of the flow in the inner area which contains these "true" jets and excludes the coastal current.

Visual comparison between the fields in the experiments with different forcing (Fig. 3) shows that the scales of the turbulent eddies generated by the forcing are noticeably different. The eddies in the thermal experiment are smaller, which indicates smaller $R_{\mathrm{d}}$. Another difference is perhaps more significant in distinguishing between the forcing mechanisms. The flow with saline forcing is characterised by thin filaments rather than circular eddies. In fact, eddies appear only as a result of breaking of the filaments and do not have a very long lifetime. The filaments are created by the baroclinic instability; they protrude in the radial (meridional) direction. 


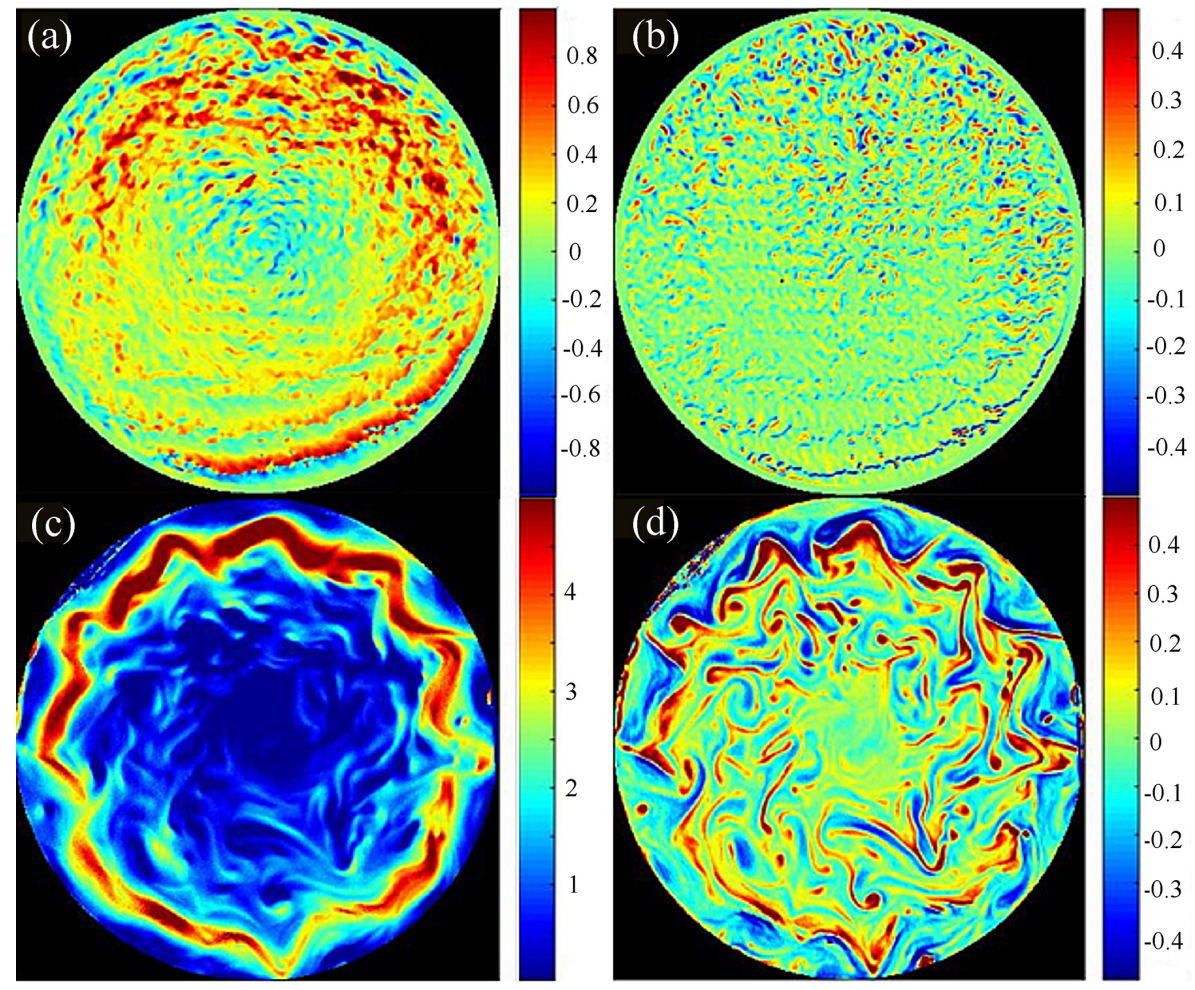

Figure 3. Flows generated by thermal forcing at $t=280 \mathrm{~s} \mathrm{(a,} \mathrm{b)} \mathrm{and} \mathrm{by} \mathrm{saline} \mathrm{forcing} \mathrm{at} t=150 \mathrm{~s}$ (c, d). (a) and (c) show the $x$ component of velocity (azimuthal velocity) $u$, while (b) and (d) show the dimensionless relative vorticity, $\zeta / f_{0}$. The salinity difference between the layers for the experiment with saline forcing $(\mathbf{a}, \mathbf{b})$ is $S=30 \mathrm{ppt}$. The centre of the tank corresponds to the North Pole of the polar $\beta$-plane.

We hypothesise that these filaments are the manifestation of the so-called "noodles" which are the primary mode of the instability of the baroclinic flow (Berloff et al., 2009a, b).

\subsection{Energy spectra in wavenumber space}

Herein we describe the results of the spectral analysis of the flows. For a circular domain such as our tank, it is perhaps more natural to use polar coordinates for the purpose of spectral decomposition. Afanasyev and Wells (2005) used Fourier-Bessel transform to obtain two-dimensional energy spectra of the polar $\beta$-plane turbulence in the space of azimuthal and radial wavenumbers and then to obtain onedimensional spectrum by sorting data in terms of a polar analogue of the Cartesian isotropic wavenumber. However, it is easier to perform digital Fourier decomposition in the Cartesian coordinates because fast Fourier transform routines can be used. Moreover, usage of Cartesian coordinates and a conventional $\beta$-plane (rather than a quadratic polar $\beta$ plane) simplifies the further comparison with available theory. For these reasons here we introduce local Cartesian coordinates $(x, y)$ centred at the reference radius $r_{0}=25 \mathrm{~cm}$ such that the $x$ axis is directed to the east, $x=r_{0} \theta$ (where $\theta$ is the polar angle), and the $y$ axis is directed to the north, $y=r_{0}-r$. For the spectral analyses we chose a domain of half width $17 \mathrm{~cm}$ centred at the reference radius $r_{0}=25 \mathrm{~cm}$ such that the polar part of the tank, where $\beta$-plane approximation is inappropriate, and the wall area, where the coastal jet dominates, were excluded.

The AIV technique gives velocity field on a regular rectangular grid covering the entire area of the tank. The velocity vector field was interpolated onto the local Cartesian coordinate system and then projected to the eastward and northward directions to obtain zonal and meridional velocity components. Discrete Fourier transform of these velocity components then gives velocity $\boldsymbol{u}\left(k_{x}, k_{y}\right)$ in the wavenumber space $\left(k_{x}, k_{y}\right)$. The two-dimensional energy spectrum is given by

$E\left(k_{x}, k_{y}\right)=\frac{1}{2}\left|\boldsymbol{u}\left(k_{x}, k_{y}\right)\right|^{2}$.

Figure 4 shows two-dimensional spectra in the experiments with thermal and saline forcing. The spectra are measured in the beginning of both experiments when the spectral signature of the forcing is still strong, and at a later stage, when the spectra have reached a certain "saturation" and the turbulent cascades are developed. The spectral properties of forcing or background turbulent flow can be inferred from the initial spectra. In the experiment with thermal forcing the eddies generated by thermal plumes are quite small; their scale can be estimated from the value of wavenumber $k \approx 2.5 \mathrm{~cm}^{-1}$ (outer ring in Fig. 4a) to be $\sim 2.5 \mathrm{~cm}$. Here $k=\left(k_{x}^{2}+k_{y}^{2}\right)^{1 / 2}$ is the isotropic wavenumber. These eddies are initially con- 

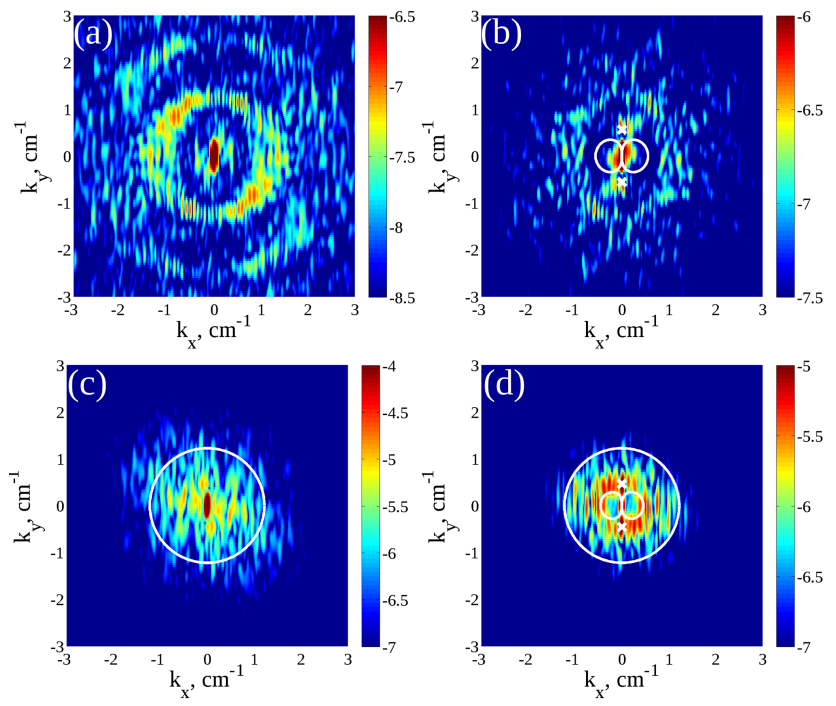

Figure 4. Energy spectra in the wavenumber space $\left(k_{x}, k_{y}\right)$ for the experiments with thermal (a, b) and saline (c, d) forcing:

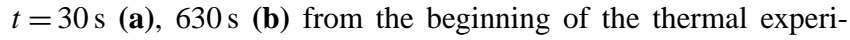
ment; $t=10 \mathrm{~s} \mathrm{(c),} 722 \mathrm{~s} \mathrm{(d)} \mathrm{after} \mathrm{the} \mathrm{fresh} \mathrm{water} \mathrm{source} \mathrm{stopped}$ in the experiment with saline forcing. Colour shows energy in logarithmic scale. Black circles in (c) and (d) show $R_{\mathrm{d}}^{-1}$. Black crosses in (b) and (d) represent the Rhines scale wavenumber (5), while the dumbbell-shaped curve is given by Eq. (6).

centrated along the heating wires. The separation of the heating wires $(\sim 4.5 \mathrm{~cm})$ determines a wavenumber $k \approx 1.4 \mathrm{~cm}^{-1}$ where energy concentration is observed as well (inner ring in Fig. 4a). In the experiment with saline forcing the initial spectrum is determined by the baroclinic instability which is sustained in the two-layer system even when the actual forcing (the injection of fresh water) is stopped. The energy is distributed in a wide range of wavenumbers, but is mainly contained within a circle which corresponds to the reciprocal of the radius of deformation, $R_{\mathrm{d}}^{-1}=1.2 \mathrm{~cm}^{-1}$. This is in agreement with the prediction of the Phillips model (Phillips, 1951) of baroclinic instability. The model predicts that all perturbations of wavenumber $k<R_{\mathrm{d}}^{-1}$ are unstable, with the most unstable (before non-linear saturation) wavenumber being $k=0.64 R_{\mathrm{d}}^{-1}$. Thus the initial spectra for both experiments confirm our initial observation that the forcing scale is smaller in the experiment with the thermal forcing. The spectra measured in much later times in both experiments (Fig. 4b and d) show that energy cascades towards smaller wavenumbers (larger scales). The distribution of energy in the wavenumber space also becomes more anisotropic; the energy flows towards the $k_{y}$ axis (zonal modes, $k_{x}=0$ ).

The inverse energy cascade is a well-known phenomenon in two-dimensional turbulence; energy is transferred from small scales (large $k$ ) to large scales (small $k$ ). In the presence of $\beta$-effect, this scenario is modified. The pioneering work by Rhines (1975) demonstrated that there is a certain
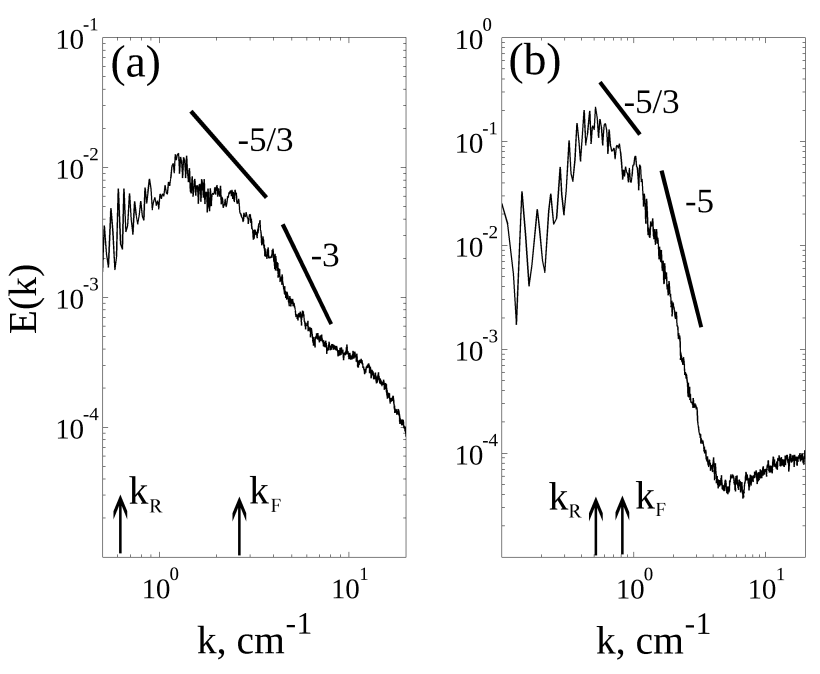

Figure 5. The one-dimensional energy spectra in log-log scale for the experiments with thermal (a) and saline (b) forcing: $t=630 \mathrm{~s} \mathrm{(a),} 722 \mathrm{~s} \mathrm{(b)} \mathrm{(as} \mathrm{in} \mathrm{Fig.} \mathrm{4b} \mathrm{and} \mathrm{d).}$

scale, now known as the Rhines scale, which separates the large-scale motions where $\beta$-effect dominates from a smallscale turbulence. A wavenumber corresponding to the Rhines scale is given by

$k_{R}=\sqrt{\beta / U_{\mathrm{rms}}}$,

where $U_{\text {rms }}$ is root-mean-square velocity. On large scales Rossby wave elasticity is important and the flow becomes strongly anisotropic as the (linear) dispersion relation for Rossby waves suggests. The anisotropy is manifested by the appearance of zonal jets. The wavenumber $k_{R}$ is also widely used as an estimate for the meridional wavenumber of an arrangement of zonal jets. It was shown to work well in different circumstances including flows on gas giants or flows in the laboratory, although different modifications of the Rhines scale were discussed as well. The values of $k_{R}$ for each experiment are indicated by crosses on the $y$ axis in Fig. $4 b$ and $\mathrm{d}$.

To extend the Rhines' argument to two dimensions one can equate the frequency of turbulent eddies to that of Rossby waves to obtain (Vallis and Maltrud, 1993) a dividing line in the form

$k_{8}=\sqrt{\frac{\beta \cos \theta}{U_{\mathrm{rms}}}}$,

where $\theta$ is the polar angle in the wavenumber space, $\theta=\arctan \left(k_{y} / k_{x}\right)$. The line given by Eq. (6) resembles a figure-of-eight or a dumbbell and is shown in Fig. $4 \mathrm{~b}$ and d. Note that values of $U_{\text {rms }}$ are not given by theory but have to be measured in the experiment. Here we used the rms values of the $y$ component of velocity instead of the total velocity in order to avoid the mean flow in the azimuthal direction which 
occurred to a different extent in both of the experiments. Since Rhines' theory assumes isotropic small-scale turbulence, it seems that the $y$ component of velocity represents a better measure of turbulence in our case. Spectra in Fig. 4b and $d$ show that the turbulent cascade of energy due to nonlinear interaction of modes does not continue in the isotropic manner within the area bounded by the $k_{8}$ line. In fact, there is little energy inside this area. The energy follows instead a (linear) Rossby wave dispersion relation flowing along the $k_{8}$ line towards the $k_{y}$ axis and concentrating there. It is eventually dissipated by friction which in our case is mainly the Ekman bottom friction which acts on all scales. The distribution of zonal energy indicates that the isotropic Rhines scale is indeed a reasonable measure of the jet wavenumber in both experiments.

To study general spectral characteristics of the turbulent cascade without regard to the anisotropy one can average energy over the polar angle $\theta$ in the wavenumber space. As a result, one obtains a one-dimensional energy spectrum which is defined as $E(k)=2 \pi \boldsymbol{k}\langle E(k)\rangle$, where $\boldsymbol{k}=\left(k_{x}, k_{y}\right)$ and the average is over $|\boldsymbol{k}|=k$. Figure 5 shows the one-dimensional spectra for our two experiments. Theory of two-dimensional turbulence without $\beta$-effect predicts the existence of the dual cascade such that energy cascades to large scales in the range $k<k_{\mathrm{F}}$ with a Kolmogorov type spectral slope $-5 / 3$ and to small scales in the range $k>k_{\mathrm{F}}$ with a slope -3 . Here $k_{\mathrm{F}}$ is the forcing wavenumber where energy is injected into the system. The former range is called the energy range, while the latter is called the enstrophy range. The forcing wavenumber can be estimated to be $k_{\mathrm{F}} \approx 2.5 \mathrm{~cm}^{-1}$ in the experiment with thermal forcing and $k_{\mathrm{F}}=0.64 R_{\mathrm{d}}^{-1} \approx 0.8 \mathrm{~cm}^{-1}$ in the experiment with saline forcing. These values mark the boundary between the energy and enstrophy ranges in both experiments. Figure 5 shows that the spectral slope does change in the vicinity of the estimated forcing wavenumbers that confirms that these estimates are meaningful. In both experiments, a $-5 / 3$ slope was observed in the energy range; in the first experiment (thermal forcing) this range was longer than that in the second one (saline forcing). In the enstrophy range the -3 slope was observed in the first experiment (thermal forcing), while the second experiment (saline forcing) showed a much steeper slope. The presence of the -3 slope might be another consequence of the mostly barotropic (and two-dimensional) character of the flow in the first experiment. Indeed, the flow in this experiment is convectively unstable and mixing is significant. As a result, the fluid is not significantly stratified and the flow must be mainly barotropic. The steeper slope in the second experiment is, perhaps, due to the fact that this two-layer, statically stable flow is significantly baroclinic. Note that a steeper than -3 slope was also measured in the experiments with shallow water, $f$-plane turbulence by Afanasyev and Craig (2013) and in the numerical simulations by Yuan and Hamilton (1994).
The estimates of the Rhines wavenumber (accidently) give similar values for both experiments, $k_{R} \approx 0.45 \mathrm{~cm}^{-1}$. This value indicates the place for zonal jets in the spectral cascade. The maximum energy achieved by the cascade in the second experiment roughly corresponds to the Rhines wavenumber, which indicates that zonal motions should possess a significant portion of the total energy. In the first experiment, the cascade does not quite reach the Rhines wavenumber. Note that the Rhines wavenumber does not stop the cascade to even lower wavenumbers; the cascade is just redirected towards zonal motions.

\section{Conclusions}

In our experiments we observed the formation of zonal jets in the experiments where flows were forced using two different methods. Perhaps the main difference between the forcing was that the heater at the bottom created convectively unstable vertical temperature distribution which resulted in smallscale convective plumes. Vertical mixing must be significant in this system and the fluid remained mainly unstratified. The large-scale flow in this experiment is then approximately barotropic, although the nature of forcing is baroclinic. In the second experiment, on the other hand, we created statically stable two-layer stratification. The flow was baroclinic to a significant degree. Since this system was unstable with respect to baroclinic instability, the instability was a source of small-scale turbulence. Thus in both cases some small-scale turbulence was created, but in the former experiment the flow was mainly barotropic, while in the latter it was mainly baroclinic.

In spite of this significant difference between the flows in our two experiments, we observed a definite universality in their spectral dynamics. The energy cascaded from small scales to larger scales and towards zonal motions. The twodimensional spectra demonstrated that this cascade is in reasonable agreement with the Rhines theory. One-dimensional spectra of energy reliably demonstrated the existence of the energy interval with the $-5 / 3$ slope. Note that although in our experiments one can infer the direction of the energy cascade from the form of the spectrum assuming that the forcing wavenumber is known, direct evidence of the cascade direction can only be provided by the analyses of the spectral energy flux. Such evidence was provided for shallow water rotating turbulence (without $\beta$-effect) in the laboratory investigation by Afanasyev and Craig (2013). The analysis of the energy flux for the large-scale oceanic turbulence by Scott and Wang (2005) revealed the existence of the inverse cascade in agreement with the two-dimensional turbulence theory. However, the interplay between barotropic and baroclinic modes and the extent to which each mode contributes to the cascade in the ocean still remains a subject of research. The analysis of the flux for the experiments reported here is yet to be done and will be reported elsewhere. 
Acknowledgements. The authors are grateful to Alexander Slavin for his help with one of the experiments. Y. D. Afanasyev is supported by the Natural Sciences and Engineering Research Council of Canada. Experimental data are available on request from Y. D. Afanasyev.

Edited by: J. M. Redondo

Reviewed by: W.-G. Fruh and one anonymous referee

\section{References}

Afanasyev, Y. D. and Craig, J. D. C.: Rotating shallow water turbulence: experiments with altimetry, Phys. Fluids, 25, 106603, doi:10.1063/1.4826477, 2013.

Afanasyev, Y. D. and Wells, J.: Quasi-two-dimensional turbulence on the polar beta-plane: Laboratory experiments, Geophys. Astrophys. Fluid Dyn. 99, 1-17, 2005.

Afanasyev, Y. D., Rhines, P. B., and Lindahl, E. G.: Velocity and potential vorticity fields measured by altimetric imaging velocimetry in the rotating fluid, Exp. Fluids, 47, 913-926, 2009.

Afanasyev, Y. D., O'Leary, S., Rhines, P. B., and Lindahl, E. G.: On the origin of jets in the ocean, Geophys. Astrophys. Fluid Dynam., 106, 113-137, 2011.

Bastin, M. E. and Read, P. L.: A laboratory study of baroclinic waves and turbulence in an internally heated rotating fluid annulus with sloping endwalls, J. Fluid Mech., 339, 173-198, 1997.

Bastin, M. E. and Read, P. L.: Experiments on the structure of baroclinic waves and zonal jets in an internally heated rotating cylinder of fluid, Phys. Fluids, 10, 374-389, 1998.

Berloff, P., Kamenkovich, I., and Pedlosky, J.: A model of multiple zonal jets in the oceans: dynamical and kinematical analysis, J. Phys. Oceanogr., 39, 2711-2734, 2009a.

Berloff, P., Kamenkovich, I., and Pedlosky, J.: A mechanism of formation of multiple zonal jets in the oceans, J. Fluid Mech., 628, 395-425, 2009b.

Centurioni, L. R., Ohlmann, J. C., and Niiler, P. P.: Permanent meanders in the California Current System, Phys. Oceanogr., 38, 1690-1710, 2008.

Collin de Verdiere, A.: Mean flow generation by topographic Rossby waves, J. Fluid Mech., 94, 39-64, 1979.

Fernando, H. J. S., Chen, R. R., and Boyer, D. L.: Effects of rotation on convective turbulence, J. Fluid Mech., 228, 513-547, 1991.

Griffiths, R. W. and Linden, P. F.: The stability of buoyancy-driven coastal currents, Dynam. Atmos. Oceans, 5, 281-306, 1981.

Hide, R. and Mason, P. J.: Sloping convection in a rotating fluid, Adv. Phys., 24, 47-99, 1975.

Ivanov, L. M., Collins, C. A., and Margolina, T. M.: System of quasi-zonal jets off California revealed from satellite altimetry, Geophys. Res. Lett., 36, L03609, doi:10.1029/2008GL036327, 2009.

Kraichnan, R.: Intertial ranges in two-dimensional turbulence, Phys. Fluids, 10, 1417-1423, 1967.

Marcus, P. S. and Lee, C.: A model for eastward jets in laboratory experiments and planetary atmospheres, Phys. Fluids, 10, 14741489, 1998.
Mason, P. J.: Baroclinic waves in a container with sloping endwalls, Philos. T. Roy. Soc. Lond. A, 278, 397-445, 1975.

Matulka, A. M. and Afanasyev, Y. D.: Zonal jets in equilibrating baroclinic instability on the polar beta-plane: experiments with altimetry, J. Geophys. Res.-Oceans, 120, 6310-6144, doi:10.1002/2015JC011083, 2015.

Maximenko, N. A., Bang, B., and Sasaki, H.: Observational evidence of alternating zonal jets in the world ocean, Geophys. Res. Lett., 32, L12607, doi:10.1029/2005GL022728, 2005.

Maximenko, N. A., Melnichenko, O. V., Niiler, P. P., and Sasaki, H.: Stationary mesoscale jet-like features in the ocean, Geophys. Res. Lett., 35, L08603, doi:10.1029/2008GL033267, 2008.

Maxworthy, T. and Narimousa, S.: Unsteady, turbulent convection into a homogeneous, rotating fluid, with oceanographic applications, J. Phys. Oceanogr., 24, 865-887, 1994.

Phillips, N. A.: A simple three-dimensional model for the study of large scale extra tropical flow pattern, J. Meteorol., 8, 381-394, 1951.

Pratt, W.: Digital Image Processing, Wiley-Interscience, Hoboken, New Jersey, 2007.

Read, P. L., Yamazaki, Y. H., Lewis, S. R., Williams, P. D., Wordsworth, R., Miki-Yamazaki, K., Sommeria, J., Didelle, H., and Finchman, A.: Dynamics of convectively driven banded jets in the laboratory, J. Atmos. Sci., 64, 4031-4052, 2007.

Read, P. L., Jacoby, T. N. L., Rogberg, P. H. T., Wordsworth, R. D., Yamazaki, Y. H., Miki-Yamazaki, K., Young, R. M. B., Sommeria, J., Didelle, H., and Viboud, S.: An experimental study of multiple zonal jet formation in rotating, thermally driven convective flows on a topographic beta-plane, Phys. Fluids, 27, 085111, doi:10.1063/1.4928697, 2015.

Rhines, P. B.: Waves and turbulence on a beta-plane, J. Fluid Mech., 69, 417-443, 1975.

Rhines, P. B., Lindahl, E. G., and Mendez, A. J.: Optical Altimetry: A new method for observing rotating fluids with application to Rossby waves on a polar beta-plane, J. Fluid Mech., 572, 389412, 2006.

Scott, R. B. and Wang, F.: Direct Evidence of an Oceanic Inverse Kinetic Energy Cascade from Satellite Altimetry, J. Phys. Oceanogr., 35, 1650-1666, doi:10.1175/JPO2771.1, 2005.

Slavin, A. G. and Afanasyev, Y. D.: Multiple zonal jets on the polar beta plane, Phys. Fluids, 24, 016603, doi:10.1063/1.3678017, 2012.

Smith, C. A., Speer, K. G., and Griffiths, R. W.: Multiple zonal jets in a differentially heated rotating annulus, J. Phys. Oceanogr., 44, 2273-2291, 2014.

Sommeria, J., Meyers, S. D., and Swinney, H. L.: Laboratory simulation of Jupiter's great red spot, Nature, 331, 683-689, 1988.

Sommeria, J., Meyers, S. D., and Swinney, H. L.: Laboratory model of a planetary eastward jet, Nature, 337, 58-61, 1989.

Vallis, G. K. and Maltrud, M. E.: Generation of mean flows and jets on beta plane and over topography, J. Phys. Oceanogr., 23, 1346-1362, 1993.

Whitehead, J. A.: Mean flow driven by circulation on a $\beta$-plane, Tellus, 27, 358-364, 1975.

Wordsworth, R. D., Read, P. L., and Yamazaki, Y. H.: Turbulence, waves and jets in a differentially heated rotating annulus experiment, Phys. Fluids, 20, 126602, doi:10.1063/1.2990042, 2008. 
Yuan, L. and Hamilton, K.: Equilibrium dynamics in a forceddissipative f-plane shallow water system, J. Fluid Mech., 280, 369-394, 1994.
Zhang, Y. and Afanasyev, Y. D.: Beta-plane turbulence: Experiments with altimetry, Phys. Fluids, 26, 026602, doi:10.1063/1.4864339, 2014. 